EGU2020-18985

https://doi.org/10.5194/egusphere-egu2020-18985

EGU General Assembly 2020

(c) Author(s) 2020. This work is distributed under

the Creative Commons Attribution 4.0 License.

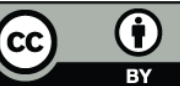

\title{
The role of cover and pattern of installed resource sinks in the recovery of degraded patchy drylands
}

\author{
Angeles Garcia Mayor, Paul Berghuis, Max Rietkerk, and Mara Baudena \\ University of Utrecht, Copernicus Insitute of Sustainable Development, Netherlands (a.garciamayor@uu.nl)
}

A low-cost restoration in patchy drylands aiming at recovering the ability of the ecosystem to capture and store water and nutrients is the installation of obstructions to break runoff pathways and retain these resources (hereafter, resource sinks). Field works in drylands worldwide have studied how the effectiveness of this action depends on the materials used to build the obstructions. However, the spatial pattern attributes of the resource sinks can also affect the effectiveness of the restoration and has not yet been investigated. In this work, we cover this knowledge gap by using a well-known dryland model to investigate how different initial amounts of cover and spatial distribution of installed resource sinks (i.e., random vs. regular) affects the recovery of the system. In agreement with field-work studies, our model results confirm that the installation of resource sinks can restore degraded drylands that are not able to recover naturally. More importantly, we found that a very small cover of resource sinks was sufficient to trigger the recovery of vegetation, while a high cover could lead to a complete failure of vegetation recovery. This was found for both random and regular distributions of resource sinks. However, a distribution similar to that of vegetation in the reference healthy system (i.e., regular distribution in our study system) was more effective: higher plant densities were reached for a given initial cover of resource sinks. Given the high efficiency of low covers of resource sinks suggested by our work, combined with the low-cost materials needed, the installation of resource sinks in severely degraded drylands has the potential to be a key contributor to the large restoration efforts needed to achieve land-degradation neutrality in the coming decades, particularly in developing countries.

How to cite: Garcia Mayor, A., Berghuis, P., Rietkerk, M., and Baudena, M.: The role of cover and pattern of installed resource sinks in the recovery of degraded patchy drylands, EGU General Assembly 2020, Online, 4-8 May 2020, EGU2020-18985, https://doi.org/10.5194/egusphereegu2020-18985, 2020 